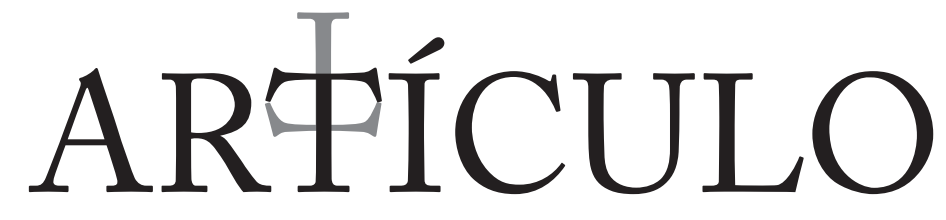

\title{
BIBLIOGRÁFICO
}

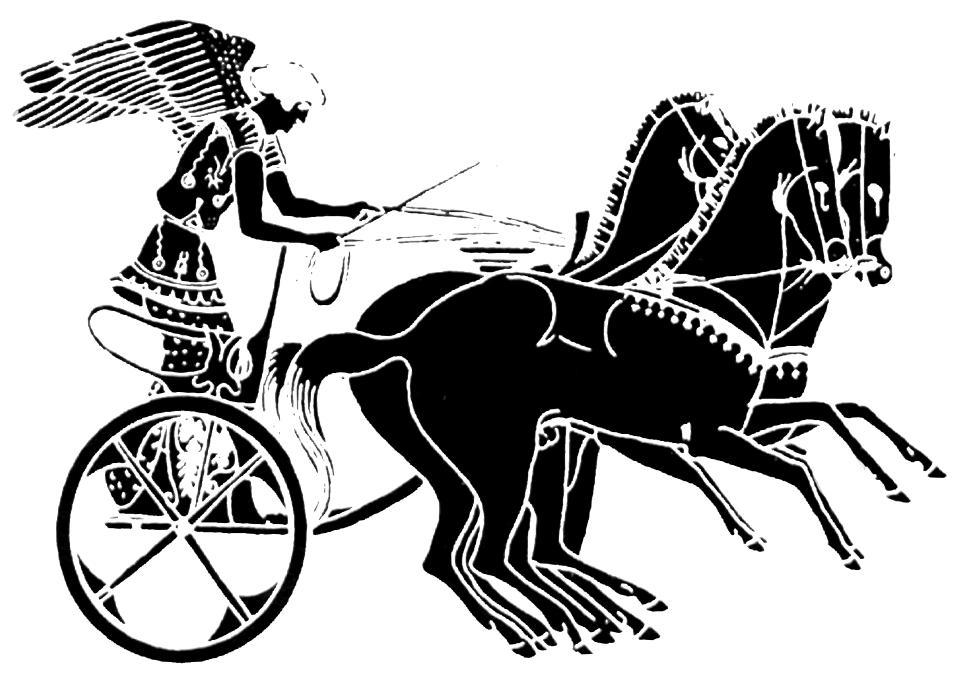



Tras haber publicado ya, en esta misma revista (10/2006 y 11/2007), las dos primeras unidades, cierro el microsistema de textos míos sobre Coseriu en un determinado campo (enseñanza de idiomas y traducción) con la tercera y última entrega.

PALABRAS CLAVE: bibliografía, lengua española, traducción, historiografía lingüistica.

\section{Trabajos de Eugenio Coseriu, en lengua española, sobre la traducción}

\section{y su entorno}

JosÉ PoLO

Universidad Autónoma de Madrid
Works in spanish by Eugenio Coseriu on translation and related topics

After publishing the first two units in this same journal (10/2006 and 11/2007), I am closing the microsystem of my own texts about Coseriu in a specific field (foreign language teaching and translation) with the third and last set.

KEYWORDS: bibliography, Spanish language, translation, linguistic historiography. 


\section{O-I}

La presente contribución se inserta en el proyecto 104 EFI2008-04605/FILO (Lingüistica de Eugenio Coseriu y lingǘstica coseriana) del Ministerio de Ciencia e Innovación (Subdirección de Proyectos). Se sitúa en la fase preparatoria de un gran espacio que desembocará en una edición crítica de una vieja y trascendental obra (inédita como totalidad proveniente del cauce «escritura», no en cuanto transmisión oral en cursos varios) del maestro: El problema de la corrección idiomática.

\section{0-2}

Por otra parte, constituye esta unidad de ahora el tercero y último elemento de una trilogía cuyas dos primeras entregas fueron: I) «Estudios de Eugenio Coseriu, en lengua española, sobre aprendizaje/enseñanza de idiomas y gramática contrastiva», en Trans. Revista de Traductología (Universidad de Málaga), Io/2006, págs. 85-98; 2) «Pautas conceptuales, terminológicas y estilísticas para la traducción de mis obras: esbozo» (dispuesto para la imprenta por José Polo), en la misma revista acabada de nombrar, II/2007, págs. 247-257.

\section{0-3}

Dejo fuera del repaso en marcha — pues poseen personalidad propia y merecen asedios individualizados, además de que, cuando menos, se triplicaría la extensión del recorrido actual - tres frentes, igualmente de mucho interés, a saber: I) las traducciones, del español al italiano, principalmente de poesía, realizadas por nuestro autor (textos de Antonio Machado, Gerardo Diego, etc.); 2) diversas traducciones literarias al italiano desde lenguas varias; 3) el fomento de traducciones de estudios de lingüística y de estética llevadas a cabo en Montevideo, en determinado período de su magisterio his-

pánico, para seminarios y cursos, así como su propia labor traductora en más de una ocasión; 4) material «infraestructural»: los cuadernos con mis notas del examen, junto a la palabra de Coseriu, de los miles de unidades de los fondos de su archivo (lo que, para mi uso particular, bauticé como «Los veranos de Tubinga: r996-2002»). Así que me he limitado, por razones comprensibles de relativamente ágil y perentorio fruto, a ordenar lo que se halla suelto, en letra de molde (y etapa previa de conferencias), por esos mundos de Dios.

\section{0-4}

Finalmente, anuncio que, en coherencia con el espíritu y materialidad de esta serie, no me adentraré en lo publicado sobre esta zona por Coseriu en lenguas distintas de la española, aunque, para no privar al estudioso de los datos de origen, de la línea de transmisión de un determinado trabajo, apuntaré lo imprescindible como guía necesaria.

\section{DOS CALAS HISTORIOGRÁFICAS}

O

Sin duda alguna, una visión completa del conjunto de la obra de Coseriu nos daría un cúmulo notable de atisbos, referencias, etc., de real o virtual connotación historiográfica. Aquí, sin embargo, me voy a limitar a dos perfectamente discernibles y que representan, claro está, un nivel metalingüístico último que permitiría su presencia como cierre del presente desfile. He preferido, no obstante, situarlo aquí a manera de estímulo visible de tiempo ha en el pensamiento coseriano y que «explica» el que él le haya prestado una atención permanente y sistemática a esta clase de asuntos (hecho favorecido, entre otras razones, por su reconocido dominio de lenguas...). 


\section{A}

I. «Panorama de la lingüística iberoamericana (I940-I965)», cap. XII, págs. 264-364, en Tradición y novedad en la ciencia del lenguaje. Estudios de historia de la lingüistica, Gredos, Madrid, I977. Constituye el texto original del que se había traducido la versión inglesa en el vol. IV, I968, págs. 5-62, de Current Trends in Linguistics (Mouton, La Haya-París). El segmento temático 4.I.I.I, págs. 302-304, se inicia con estas palabras: «La primera vía de la información lingüística general en Iberoamérica es, sin duda, la de las traducciones»; luego viene el sucinto, pero esclarecedor, desarrollo informativo de tan incisiva y exacta aseveración, con nombres clásicos de autores y de traductores-anotadores, etc. El último párrafo (págs. 303-304), en el que omito las notas, reza así:

Lamentablemente, las traducciones no realizadas o dirigidas por especialistas presentan dos inconvenientes: por un lado, suelen ser deficientes, sobre todo en lo referente a la terminología lingüística (así, entre otras, la traducción de[...]); por el otro, no obedecen a criterios seguros en la selección, lo cual encierra un peligro bastante grave, pues, para los desprevenidos, el hecho de que una obra haya sido traducida constituye certificado de excelencia.

2. Y dentro del 6.3.5, págs. 340-342, hay unas líneas (pág. 34I) cuyo comienzo es: «Sobre el problema de la traducción, la contribución teórica más importante publicada en Iberoamérica es[...]»; y, al igual que en el anterior espacio, aparecen trascendentes autores con sus respectivas obras.

\section{B}

3. Nuestro autor había publicado en alemán, en I971, el trabajo que luego fue traducido al español (por Marcos Martínez Hernández) con el título de «Acerca de la teoría del lenguaje de Luis Vives», cap. II, págs. 62-85, en el volumen atrás, ficha I, mencionado. Podemos decir que esta contribución le crea el entorno a otro estudio (originalmente también en alemán y de I97I), que es: «Vives y el problema de la traducción», en dicha obra, III, págs. 86-Io2 (el mismo traductor). En págs. IOI-IO2 hay una larga nota, texto posterior a la de la edición alemana, en el que el maestro replica a unas observaciones de G. Folena en artículo de i973. Transcribo las últimas líneas:

Es cierto que la historia de la teoría de la traducción no puede hacerse sin tomar en cuenta las contribuciones del humanismo italiano, pero esto no tiene nada que ver con la finalidad de mi ensayo y no quita a Vives el mérito de ser, en un aspecto esencial, precursor de la teoría moderna del traducir.

4. Ambos estudios coserianos fueron luego reunidos en el opúsculo Dos estudios sobre Juan Luis Vives, Asociación de Lingüística y Filología de la América Latina (Cuadernos de Lingüística, 4) México, D. F., I978; véase, por ejemplo, la reseña de Tudora ŞAndru-Olteanu, en Anuario de Letras, I9/198I, págs. 335-339. Sobre el primero de esos trabajos, el de carácter general, véase, entre otras referencias posibles, José María Bernardo Paniagua, «Hacia una teoría del lenguaje en Luis Vives» (naturalmente, con la presencia trascendente de Coseriu), en III Congrés d'Història i Filologia de la Plana. Homenatge en Josep Trenchs Odena (celebrado en 1992), Nules [Castellón], I994, págs. 257-270. Cabría, naturalmente, llevar a cabo una búsqueda exhaustiva de las referencias al segundo de los estudios, traducción, sobre Vives. Me limitaré, sin embargo, cual mera llamada de atención, a dos especímenes: I)Valentín García Yebra, 
«Un teórico de la traducción hispanoflamenco» (conferencia en la Universidad de Lovaina: 29 de abril de 1987), en su libro Traducción: bistoria y teoría, Gredos, Madrid, I994, págs. I7I-I86. En I72-I73 se pone de relieve la importancia del artículo traductológico de Coseriu; y en nota, en la segunda de esas páginas, podemos leer:

Con posterioridad a la redacción de esta conferencia publicó Francisco Calero, en [...], en que no sólo discrepa en algún punto de la interpretación de Luis Vives por Coseriu, sino también de algunas ideas de Luis Vives sobre la traducción; censura especialmente la traducción de Luis Vives por Lorenzo Riber [Aguilar, Madrid, I948].

5. El trabajo mencionado, cuyos datos he omitido en la cita anterior, es, en efecto, el de Francisco Calero «Sobre la teoría de la traducción de Luis Vives», en Homenatge a José Esteve Forriol, Universitat de València, I990, págs. 37 (portadilla) y, texto, 39-46 (el apéndice comprende las tres últimas páginas y en él se presenta una nueva traducción del capítulo I2 del libro III de Rethoricae, sive de recte dicendi ratione libri tres). Coseriu se halla mencionado varias veces en págs. 39 y 4I, una sola vez en $42 \mathrm{y}$, bibliográficamente, con sus trabajos sobre Vives, en 43 (no hace acto de presencia Juan José Calvo: véase enseguida ficha 6). Citaré el comienzo del primer párrafo: «Hay que agradecer al gran lingüista E. Coseriu el haber llamado la atención de los investigadores sobre la teoría lingüística de Vives, así como sobre su teoría de la traducción, ya que ambas eran completamente desconocidas incluso para los especialistas[...]; en este trabajo me voy a fijar solamente en la problemática de la traducción del gran humanista valenciano, ya que tengo algunos desacuerdos con la interpretación dada por Coseriu». No me consta ningún texto del maestro en el que haya podido hacerse cargo de los planteamientos del profesor Calero (lo confirmo, además, consultando con el investigador acabado de mencionar), aunque convendrá fijarse en estudios posteriores a I990 por si hubiese alguna referencia a dicho texto. Y con respecto al traductor de Vives mencionado en la ficha anterior, conviene ver, del mismo profesor Calero, su trabajo «Traducir a Vives: elogio crítico de Lorenzo Riber», en Cuadernos de Filología Clásica. Estudios Latinos (Universidad Complutense, Madrid), I5/I998, págs. 529-539.

6. Pero unos cuantos años antes había publicado Juan José CALvo su artículo «Juan Luis Vives: ¿un pionero de la traductología moderna? Apuntes sobre una discrepancia», en Estudis en memòria del professor Manuel Sanchis Guarner: estudis de llengua i literatura catalanes, Universitat de València, I984, II, págs. 69-78. Se refiere a las diferencias interpretativas entre Eugenio Coseriu y Gianfranco Folena (véase atrás ficha 3), pero, claro está, no puede tener en cuenta la respuesta del autor rumano-uruguayo en el volumen de 1977 .

\section{SEGMENTOS TEXTUALES TRADUCTOLÓGICOS}

Voy a presentar en este epígrafe referencias varias que se hallan aquí y allá en diversos estudios de Coseriu (nombre que ya no mencionaré en la ficha) no relacionados directamente con la traducción: algo así como si pretendiera colocar ante la vista del lector una muestra de lo mucho que cabría recoger examinando el conjunto de la bibliografía coseriana, recorrido este último que nos daría el índice completo de todos los segmentos textuales en los que aparece alguna idea o información atinentes al 
ámbito que ocupa nuestra atención. Digamos, en expresión familiar y lúdica, que se trata de una suerte de «aperitivo traductológico».

I. "Notas de lectura sobre un libro de Bousoño» (redactado probablemente entre 1952 y 1955), en Analecta Malacitana, XXVI-2/2003, págs. 623-644. Constituye una de las entregas de la serie "Eugenio Coseriu y su entorno. Textos originales y traducciones de una época prodigiosa (Montevideo, I95I-I963), ordenados y dispuestos para la imprenta por José Polo». Bien: el epígrafe número 7, págs. 634-636, contiene, entre los segmentos textuales $d$ y $f$, consideraciones en torno a la comprensión de las realidades histórico-culturales para entender, en una traducción, el sentido, por ejemplo, de determinados poemas.

2. «Sobre equivalencias interidiomáticas» (oralmente, I962, en congreso; en actas de congreso, I965, en español), recogido en el volumen El hombre y su lenguaje. Estudios de teoria y metodología lingüística, Gredos, Madrid, I977, dentro del capítulo V, «Quaestiones disputatae» (págs. I59-I74), I70-I7I. Cito el primer párrafo y unas líneas de lo que sigue inmediatamente (entre esas dos páginas; omito bibliografía temporal-aspectual coseriana en relación con la idea apuntada):

Es, sin duda, interesante destacar los valores propios de una lengua mediante la comparación con otras lenguas. Esto puede a menudo revelar hechos que, de otro modo, correrían el riesgo de quedar inadvertidos. Pero puede ser peligroso el querer establecer (deslindar, identificar) y describir los valores propios de una lengua mediante su traducción a otras lenguas. Así, es cierto que los «perfectos» románicos corresponden a menudo, en la traducción, al aspecto perfectivo de las lenguas eslavas; sin embargo, los respectivos valores de lengua son radicalmente diferentes. La traducción nos dice sólo que los «perfectos» románicos y el aspecto perfectivo eslavo pueden designar los mismos hechos reales: nos permite comprobar una coincidencia (parcial) en la designación; pero de ésta no puede deducirse ninguna coincidencia en la significación. Hay que cuidarse mucho de aplicar a una sistema lingüístico categorías que le son ajenas. En efecto[...].

3. En el mismo volumen, «Sobre el desarrollo de la lingüística» (en forma oral, I972), x, págs. 257-263. Interesa directamente a nuestro propósito el párrafo 3.2, pág. 262:

Los progresos y los nuevos planteamientos de la lingüística teórica y descriptiva tendrán, ciertamente, sus repercusiones en el campo de la lingüística aplicada, en particular en la teoría de la traducción (que depende de una comprensión exacta de la distinción entre designación, significado y sentido) y en la enseñanza de las lenguas (para la cual es imprescindible la misma distinción, así como la distinción correlativa entre saber elocucional, saber idiomático y saber expresivo).

4. Principios de semántica estructural, Gredos, Madrid, I977 (tr. de Marcos Martínez Hernández). Contamos con segmentos textuales referidos al hecho de la traducción en los siguientes lugares: $\mathrm{I}$ ) en $\mathrm{V}$ (originalmente, I970), 4.6/4.6.2, págs. 208-209. Transcribo el párrafo 4.6 (el planteamiento; 6.I y 6.2, ilustración junto a determinadas consideraciones metodológico-teóricas):

No menos importantes son las implicaciones que la distinción entre designación y significado tiene para la teoría y práctica de la traducción. Señalemos aquí sólo dos de ellas, que conciernen al problema de los límites de la posibilidad del traducir. A este respecto hay 
que advertir ante todo que sólo se traducen significaciones de habla (acepciones): los significados (significaciones de lengua) como tales no se traducen ni pueden traducirse, como, naturalmente, tampoco se traducen los «estados de cosas»[,] que, como tales, no son siquiera lingüísticos. La tarea propia de la traducción es, pues, la de d e si g n a r los mismos «estados de cosas» por medio de otra lengua, o sea, la de decir «lo mismo» — como significación de habla - por medio de significados en principio diferentes.

5. [La] competencia lingüistica. Elementos de la teoría del hablar (originalmente en alemán, publicación interna producto de cursos entre I983 y I985; elaborado y editado ulteriormente, I988, por Heinrich Weber; tr. de Francisco Meno Blanco), Gredos, Madrid, I992. Dentro del epígrafe 2.3.7, «Cometidos de una lingüística del hablar en general» (págs. I47-I52), aparecen importantes reflexiones sobre la teoría y la práctica de la traducción entre las páginas I49 y I5I. Transcribo el primer segmento textual (I49; he colocado directamente, hispanizando sin interpolación, coma tras la repetida fórmula /i.e./, 'id est'):

Quien traduce,[coma del original] trabaja con al menos dos lenguas desde una perspectiva práctica. Al traducir[,] no vamos de forma inmediata de configuración lingüística a configuración lingüística, i.e., directamente del significado en una de las lenguas al significado en la otra lengua, sino que averiguamos primeramente cuál es la designación, i.e., lo que se quiere decir extralingüísticamente. Una vez que hayamos identificado eso, nos preguntamos cómo puede expresarse la misma realidad extralingüística, i.e., la misma designación, en la lengua de llegada, i.e., cuál es la configuración válida para la otra lengua. Los significados, es decir[,] lo que dice una lengua particular como lengua particular, no pueden en modo alguno ser traducidos. Sólo pueden ser explicados, analizados y descritos en la gramática y en el diccionario de la lengua correspondiente. Por tanto, al traducir, lo primero son los significados de la lengua de partida,[:] se identifican las designaciones y luego se buscan en la lengua de llegada los significados que corresponden a las designaciones en el entorno textual que sea del caso [...].

\section{Finalmente, Lingüistica del texto. Intro-} ducción a la hermenéutica del sentido (edición, anotación y estudio previo de Óscar Loureda Lamas), Arco-Libros, Madrid, 2007. Una lectura muy atenta para un artículo-reseña que se publicará en tres partes en Analecta Malacitana (XXXIV-2/20II, págs. 599-633, y los dos siguientes), me ha permitido, entre otros deslindes de zonas temáticas, aislar las páginas en las cuales se habla de la traducción (metodología, teoría, práctica); son estas: 25, 52, 6I, 63, 65, 93-94, I30, I38-I39, I46-I47, I52-I54, I87, I94, I98, 205, 2I5, $230-231,279-280$ y 317 .

\section{CONFERENCIAS Y CURSILLOS SOBRE LA TRADUCCIÓN}

\section{$\mathrm{O}$}

Puesto que se trata de un terreno resbaladizo, nunca tan «estabilizado» como el de las publicaciones canónicas, no es posible presentar una lista completa - menos, exhaustiva-, pero lo que aparecerá servirá de llamada de atención y de estímulo para una futura investigación bio-bibliográfica específica al respecto. En todo caso, lo más importante a efectos de fijación textual es observar el tránsito desde la primera salida pública (generalmente, en los hábitos de Coseriu, hablando directamente o, en ocasiones, a partir de un esquema manuscrito o algo similar) a su estadio final (no siempre en 
texto revisado por su autor o cuyas pruebas de imprenta hayan sido corregidas por él), a su estadio final, decía, sea o no el último posible o aconsejable; pensemos, por ejemplo, en ediciones necesariamente críticas de algunos trabajos de Coseriu en un imprescindible proyecto de Obras Completas.

I. En el Curso Superior de Filología Española (Málaga; creado y dirigido por Manuel Alvar) de 1978 intervino Coseriu con una conferencia titulada Teoría de la traducción.

2. En la revista uruguaya Prometeo, II-5/1980, págs. 57-58, aparece la noticia sobre «Ciclo de seminarios y conferencias con motivo del trigésimo aniversario de la fundación del Departamento de Lingüística» (creado por Eugenio Coseriu). Nuestro autor dio varias conferencias y, entre ellas, Teoría y práctica de la traducción (el 8 de noviembre a las ir de la mañana).

3. Por fotocopia de carta que en su día me facilitó quien la firma, Emilio Lorenzo (director del Curso de Extranjeros y responsable, igualmente, del Curso Superior de Filología Hispánica, Universidad Internacional Menéndez Pelayo, Santander), con fecha de 28 de marzo de I980, podemos saber (reajusto el texto ortotipográficamente):

Los tres títulos que propone me gustan mucho y no me parecería mal que los repartiera a discreción entre las cinco lecciones. Por ejemplo, I) Más allá del estructuralismo; 2) Teoría de la traducción; 3) Tipología lingüistica. Aunque si las cinco lecciones fueran de tipología, me encantarían personalmente, pues ando dándole vueltas al tema (con relación al español) y me gustaría aprender de quien tanto sabe.
Indagaciones ulteriores me permiten señalar que, en efecto, entre los días 4 y I4 de agosto pudo complacer a E. Lorenzo con varias lecciones bajo el título de El tipo lingüistico románico, pero que no desatendió (una conferencia: Teoría de la traducción) el frente que nos interesa.

4. En la sede o extensión de Valencia de la Universidad Internacional Menéndez Pelayo, entre el I2 y el r6 de septiembre de r994, se celebró, dirigido por Ángel López García, el curso Traducción y contraste lingüistico-cultural. E1 día I3 intervino nuestro autor con la conferencia Alcances y limites de la traducción, que, como se verá más adelante, fue el título de uno de los textos publicados.

5. La traducción y sus disciplinas afines fue el Curso de Verano de la UNED (Universidad Nacional de Educación a Distancia): Ávila, 3-7 de julio de I995. Coseriu disertó, el día 3, con el mismo título de la conferencia anterior (en el programa consultado, con un pequeño cambio «numérico», alcances/alcance, no sé si intencionado o «errático sin error»): Alcance y límites de la traducción.

\section{ENTREVISTAS}

Son abundantes las entrevistas hechas al Dr. Coseriu a lo largo de su vida profesional. Como siempre, sin ánimo de exhaustividad, voy a presentar tres de ellas en las que se habla de la traducción.

I. Ricardo Mairal y Pedro Santana firman «Entrevista a Eugenio Coseriu», en Cuadernos de Investigación Filológica (Universidad de La Rioja, Logroño), XVI/I-2/I990, págs. I59-I70. 
El primer párrafo reza así: «El texto que ofrecemos a continuación es el resultado de la transcripción de parte de una entrevista mantenida con el gran lingüista rumano, la cual tuvimos la ocasión de realizar con motivo de su participación en el curso El componente léxico. Enfoques funcionales y formales, organizado por la Universidad de Granada y que tuvo lugar en Almuñécar en septiembre de i990». Ante la pregunta "Ahora parece que se están introduciendo criterios de cientificidad en la lingüística en el sentido de que una gramática es buena si sirve para hacer funcionar una máquina de traducir. ¿Qué opina vd. [Vd.] de esto? ¿No se han cambiado los objetivos del análisis lingüísticos [lingüístico]? », contesta Coseriu (pág. I65):

La gramática es una actividad humana y se hace siempre con una determinada finalidad. En cuanto al método - $-\mathrm{y}$ método significa camino-[,] hay que ver qué método permite la realización de esta finalidad de la manera más sencilla y cómoda, de suerte que entonces no se trata de gramática mejor o peor, sino de gramática mejor para tal finalidad. En el caso de la gramática destinada a la traducción[,] hay que hacer la gramática destinada a esta finalidad.

Los ordenadores trabajan con sistemas binarios y hay que hacer la gramática para que la máquina pueda aplicarla. Hay, por ejemplo, que fragmentar las unidades de otro modo.

Dada la finalidad, hay que preguntarse también si la finalidad misma es una finalidad razonable y ésta que ustedes mencionan no es una finalidad razonable. No se puede hacer la gramática de una lengua para traducir textos, porque la lengua no se traduce; se traducen textos y los textos no son sólo lengua. Por tanto, es completamente absurdo hacer una gramática y pretender que sea la mejor. Ninguna gramática va a permitir jamás traducir textos como tales. Eso es imposible puesto que el texto no depende de la lengua sólo.
El hablar es una actividad mucho más compleja y se dan toda la serie de determinaciones relacionadas con el conocimiento del mundo, de los hechos culturales, etc., de suerte que lo que hay que modificar es la finalidad misma y decir que no hay que pretender una traducción de los textos reales, sino una redacción del texto real susceptible de un tratamiento automático. Sería como pedirle a un científico que ha escrito un texto en español que añada un resumen en inglés. En este caso, sería un resumen constituído [constituido] sólo por la lengua y que se pudiese traducir con facilidad.

¿Para qué necesitamos la traducción automática? No, por cierto, para traducir a Shakespeare. La máquina no hace más de lo que se le ha dado. Lo hace mucho más rápidamente que el hombre y sin error. Por eso le damos ya un texto construído [construido] de este modo. Una máquina puede traducir cifras arábigas a romanas. Muy bien. Pero no se puede uno proponer algo racionalmente imposible.

La ventaja de la máquina para el análisis incluso de los textos es que muchas veces nos hace descubrir nuestros propios errores. De ningún modo puede decirse que la gramática sea por esto mejor o peor. La gramática mejor en absoluto, sin ser una gramática para algo, es la que dice las cosas como son.

2. En El Diario de Ávila (4 de julio de I995, pág. 6; desde hace unos años, Diario de Ávila), se publica, en relación con el curso fichado atrás, 3-5, un breve texto, ilustrado, de Concha Álvarez-Claro: «El lingüista Coseriu inauguró el curso "Traducción y disciplinas afines”. Destacó la importancia de esta profesión para la convivencia y la comunicación». Tras la parte introductoria (datos sobre nuestro autor, su poliglotismo: «habla doce idiomas»; felicitación de Coseriu a la UNED por organizar dicho curso, «ya que 
para él la traducción es un elemento clave para la comunicación y[,] por tanto[,] para la convivencia», etc.), podemos leer ya el resto de la noticia:

E1 lingüista resaltó la importancia de la profesión de traductor, no sólo de textos escritos, sino también de la traducción simultánea. Coseriu afirmó que para traducir de un idioma a otro no basta con conocer las dos lenguas, sino que hay, además, que dominar las técnicas de la traducción, «saber cómo se expresan tales contenidos en tales idiomas, conocer el tema específico del que se trata», dominar los valores de esas culturas. Coseriu habló también de las dificultades de la traducción por la riqueza de los sinónimos y de la necesidad de conocer el contexto para conseguir una buena traducción. Este curso, que ha reunido a más de cuarenta y cinco alumnos, tiene como objetivo presentar un modelo traductológico integrador e interdisciplinar,[coma del original] que aglutine las contribuciones de áreas afines como la microinformática, ingeniería lingüística, etc...,[etc.,] por lo que está dirigido a investigadores y profesionales de la traducción.

3. En la realización inmediatamente posterior de los Cursos de Verano de la UNED volvió a actuar el maestro rumano-uruguayo. En El País (Madrid), desde Ávila y con la firma de S. C. (=Sección Cultural), con fecha de 6 de julio de 1996, pág. 78, se lee, tras «Cursos de Verano: UnED», el titular «Eugenio Coseriu: "Las lenguas nunca se pueden traducir,[;] los textos, sí"». Como entrada (con relieve de negrita): “A menudo se olvida que no se pueden traducir las lenguas, sino únicamente los textos. Los contenidos de una lengua no son el objeto[,] sino siempre el instrumento de una traducción", señaló Eugenio Coseriu, catedrático de la Universidad de Tubinga y uno de los fundadores del estructuralismo, que participó en el seminario Traducción y perspectivas de futuro». Luego vienen dos párrafos dedicados a su intervención académica y otra parte sobre un conferenciante distinto. Reproduzco íntegro el texto referido a nuestro autor:

El lingüista de origen rumano consideró que para traducir correctamente un texto no basta con la traslación automática de una a otra lengua, sino que lo más relevante es siempre la carga de connotaciones específicas de ambas lenguas y, en definitiva, la contextualización. «Hay un cierto conflicto entre la lingüística descriptiva y la que precisan los traductores. Éstos necesitan conocer las equivalencias de empleo de las lenguas en situaciones muy determinadas. Hay que tener siempre presente que los significados son polisémicos y que expresan un sentido». A su juicio, el «contenido del texto que se traslada no es el significado[,] sino la designación y el sentido».

Coseriu subrayó que[,] para traducir adecuadamente a una lengua[,] «hay que salirse primero de la lengua de partida. Lo determinante, por tanto, no son las lenguas, sino el empleo de las lenguas», apostilló. Ilustró las diferencias de connotaciones, por ejemplo, con los términos Sol y Luna [sol y luna, ] que en español son masculino $\mathrm{y}$ femenino, respectivamente, mientras que en alemán es al revés. «El traductor deberá privilegiar esas connotaciones de género por encima de lo puramente denotativo», señaló el lingüista, para sintetiza[r] su intervención en la siguiente frase: «La traducción tiene que identificar el sentido y para ello una lengua será siempre la clave de la otra».

\section{ARTÍ́CULOS}

I. En 1976, en un congreso en Estocolmo (Nobel Symposium 39, 6-Io de septiembre) presenta Coseriu «Falsche und richtige 
Fragestellungen in der Übersetzungstheorie»; las actas se publican en 1978: Lillebill Grähs, Gustav Korlén, Bertil Malmberg, editores, Theory and Practice of Translation, Peter Lang, Berna/Francoforte del Meno/Las Vegas, págs. 17-32; posteriormente, 1981, se reimprime la contribución de nuestro autor en W. Wilss, editor, Übersetzungswissenschaft, Darmstadt, págs. 27-47. Pero ya en 1977, antes, como vemos, de que se hubieran publicado las mencionadas actas, había aparecido la traducción al español, de Marcos Martínez Hernández, dentro del volumen, ya fichado (véase atrás 2-2) El hombre y su lenguaje, X, págs. 2I4-239: «Lo erróneo y lo acertado en la teoría de la traducción». Se verá, por la forma de confeccionar la presente ficha, que me he excedido, en relación con el espíritu de concisión que había anunciado (véase o-4), en la información respectiva. Hay una razón para ello y es que en las referidas actas (1978) se recogen los segmentos textuales de las intervenciones habidas en el coloquio subsiguiente a cada una de las respectivas exposiciones: desde la «First Discussion» hasta la «Twelfth and Concluding Discussion»; y las intervenciones de Coseriu son, como era habitual en él, enjundiosas, por no decir trascendentes. $\mathrm{He}$ aquí las páginas en las que se halla recogido el texto de dichas intervenciones (bien en estilo directo, valiéndose de la lengua alemana, bien por la voz interpuesta de los relatores en alemán, inglés o francés): 65-67, IO4, II7, I3I-I32, I45-I49, I65, 288-289, 338, 340-342, 344 y 349-350. Aporto esta información simplemente para sugerir, ya que no se hizo en su momento (cuando se tradujo su ponencia al español, pues entonces no contábamos con las actas del congreso, con el texto de las diversas intervenciones en los coloquios), para sugerir, decía, que se reúnan en un volumen todos sus estudios y notas sobre la traducción y que se aproveche la oportunidad para, creándoles el necesario contexto en forma sintética con las intervenciones de sus interlocutores, se traduzcan las de Coseriu o tal vez el volumen de actas completo con las ponencias y los coloquios (si no, al menos el conjunto de dichos textos «coloquiales»), pero que, en todo caso, no deje de verse en español ese conjunto de «interlocuciones» de nuestro autor.

2. «Los límites reales de la traducción», en Temas de lingüistica aplicada (edición de Jorge Fernández-Barrientos Martín con la colaboración de Celia Wallhead), Universidad de Granada, I995, págs. 155-I68. E1 volumen comprende una selección de las conferencias plenarias en las Jornadas Internacionales de Lingüistica Aplicada/International Conference of Applied Linguistics. Robert J. Di Pietro. In Memoriam (Granada, II-I5 de enero de 1993). Las actas se publican en forma modesta ese mismo año (edición del primero de los dos autores líneas atrás nombrados), también por la Universidad de Granada (Instituto de Ciencias de la Educación), pero de Coseriu — quizá por no hallarse aún transcrito el texto: se ve su directa procedencia oral (no es seguro que lo hubiese revisado o hubiese corregido pruebas) - solo contamos con un resumen (iredactado por su autor?; pág. II del vol. I), que transcribo:

En esta conferencia voy a hablar de los límites reales de la traducción. Se ha planteado el problema de estos límites en la teoría de la traducción,[;] incluso se han escrito libros sobre ello,[;] sin embargo, en mi opinión, estos límites no son reales, son falsos. Se entiende que el límite es causado por la estructuración diferente del sentido en una lengua comparada con otra. También hay límite porque una palabra que parece tener traducción fácil a otra lengua, como por ejemplo, bosque, traducido al alemán como Wald, puede estimular 
sentimientos distintos en la lengua meta. Estos no son los verdaderos límites, sino otros ya indicados desde la antigüedad por escritores que han tenido que vérselas con la traducción, como son Horacio y San Jerónimo. Es que las lenguas no se traducen: lo que se traduce son textos. Y dentro de los textos lo que se traduce es la realidad del sentido. Por eso, si hay conflicto entre el sentido y la designación utilizada para transmitir este sentido en una lengua para con ['con respecto a/en relación con'] otra, la lengua, siendo simplemente instrumento, es lo que hay que sacrificar por el medio de la adaptación. Para concluir, una falta de correspondencia en la relación semiótica entre lo que se dice y lo que se quiere decir a través de las lenguas es el verdadero límite de la traducción.

3. «Alcances y límites de la traducción» (en francés, «Portée et limites de la traduction»: I997-I998; en alemán: «Leistung und Grenzen der Übersetzung»:), en Lexis (Lima), XxI2/I997, págs. I63-I84. Leemos en la primera página (nota): «Versión revisada y ampliada de Abast i limits de la traducció (Lliçó inaugural del curs acadèmic 1996-97 de la Facultat de Traducció i Interpretació), Universitat Pompeu Fabra, Barcelona, I996 [i6 de octubre]». Es un texto perfectamente diferenciado del anterior y de un estilo más académico. Compárese, por otro lado, a la vista de los títulos en lenguas diferentes de la española, lo señalado atrás, 3-5, en torno a alcance/alcances.

\section{REFERENCIAS A ESOS ESTUDIOS}

\section{o}

Tampoco pretendo que esta sección agote la información al respecto: presento, simplemente, las que poseo en español (salvo una excepción comprensible) en mi archivo científico. En dos casos, a manera de contexto, haré notar la presencia de un trabajo en el que implícitamente podríamos decir que Coseriu se halla presente, se respira su ambiente «traductológico» (por tratarse de dos de sus discípulos: uno rumano y otro español). Finalmente, casi siempre, además de mencionarse en los estudios aludidos lo específico sobre la traducción en Coseriu, se citan otras investigaciones de línea no tan circunscrita, pero de las que pueden extraerse enseñanzas sobre nuestro centro de interés. Sin embargo, mi artículo va orientado nuclearmente hacia el fondo proyectado directamente sobre la traducción. Trasciendo tímidamente lo escrito en español, pero solo, como digo, en forma limitada, simplemente para lanzar al lector interesado hacia tales zonas "no hispanas» o, en algún caso (Ana Agud), «semihispana/ germano-hispana».

\section{A}

I. Sin detenerme a presentar ficha alguna, pues resultaría muy prolijo, simplemente anotaré el hecho de que en las varias obras (de recopilación o no) de Valentín García Yebra (I9I7-20Io) sobre la traducción — todas ellas en Editorial Gredos- se halla siempre muy presente el maestro Coseriu, que ha sido, por otra parte, autor de extensa y sistemática vida en dicha casa editora. Aparte del valor intrínseco de la doctrina coseriana en los temas de los que se ha ocupado y el aprecio por parte del profesor García Yebra, sin duda ha ayudado la familiaridad material de tratarse de un autor, Coseriu, «de la casa», de consulta expedita y directa.

2. Copceag, Demetrio, "Los "falsos amigos" y la teoría de la traducción», en Español Actual, 31/1976, págs. 33-38. Aunque Coseriu no se halla materialmente presente entre los autores citados, sin duda, conociendo la formación coseriana del prof. Copceag, el maes- 
tro no se halla del todo ausente. En cambio, sí resulta directamente visible en este otro trabajo del mismo estudioso rumano, a saber: «Las designaciones imaginarias en el proceso de la traducción», en Cuadernos de Traducción e Interpretación, I/I982, págs. 21-27. En efecto, arranca el Dr. Copceag del estudio «Lo erróneo y lo acertado en la teoría de la traducción» (atrás ficha 5-I). En pág. 23, tras el planteamiento del artículo, señala:

Las penetrantes observaciones de Coseriu, además de su valor intrínseco, tienen el gran mérito de estimular el pensamiento, constituyendo una fuente de inspiración para nuevas investigaciones. Precisamente así, es decir[,] como inspiradas por el enfoque coseriano del problema, deben interpretarse las consideraciones que siguen.

3. Domínguez Domínguez, J. Francisco, "Traducción y lexemática», en Estudios Humanísticos. Filología (Universidad de León), 8/1986, págs. 69-77. Aparecen no pocas referencias a trabajos varios de Coseriu (se halla presente en todas las páginas, excepto en el «medio texto» de la 77), no solo al mencionado en la ficha anterior y al de $\mathbf{I}-\mathbf{3}$ (el segundo sobre Luis Vives): véanse particularmente las páginas 7r y 76 para estos dos.

4. Cartagena, Nelson, «Funciones lingüísticas básicas y traducción», en Boletín de Filología, XXXIV/r993-1994, págs. 34-6r. Se fichan, además del repetidamente mencionado «Lo erróneo[...]», dos trabajos más no directamente traductológicos. Se le menciona en págs. 45-46, 57 (y en 59-6o, bibliografía).

5. Wotjak, Gerd, «¿De qué conocimientos debe disponer el traductor?», en Eugenio Coseriu in memoriam, II (Jesús Gerardo Martínez del Castillo, editor), Granada Lingvistica (serie
Collectae), Granada, 2004, págs. I23-I4I. Varios estudios del maestro en la bibliografía (págs. I38-I39); directamente sobre nuestro tema, el ya familiar «Lo erróneo[...]». Materialmente presente en págs. I24, I28-I29 y I32-I33.

\section{B}

6. Agud, Ana, «Über Theorie und Praxis der Übersetzung», en Energeia und Ergon: sprachliche Variation-Sprachgeschichte-Sprachtypologie. Studia in honorem Eugenio Coseriu (herausgegeben von Jörn Albrecht, Jens Lüdtke und Harald Thun), Gunter Narr Verlag, Tubinga, I988, III, págs. 351-370. Excelente trabajo en el que se menciona a Coseriu en relación con el objeto de estudio en págs. 354 y 355. Transcribiré unas líneas del primero de estos dos espacios, pero, sobre todo, quiero destacar el hermoso párrafo «discente» preliminar (pág. 35I) para entender que todo el trabajo (y otros de dicha notable estudiosa) se halla empáticamente inserto en la esplendorosa «aura docente» de este singular maestro:

I

Linguisten werden in diesem Kontext sicher die Unterscheidung von Bedeutung, Bezeichnung und Sinn [significado, designación y sentido] vermissen. Unser Jubilar [Coseriu] hat sie bekanntlich im Zusammenhang mit seiner Übersetzungstheorie weitgehend angewandt. Es muß allerdings betont werden, daß Coseriu diese Unterscheidung ausdrücklich zum Zwecke der Abgrenzung des Begriffs der "Übertragung" einführt, und zwar tut er das in bezug auf die Möglichkeit linguistischer Theoriebildung durch kontrastive Grammatiken. Ich verstehe meinen eigenen Ansatz hier als Complementär dazu.

Prof. Coseriu war mein Lehrer während der wohl wichtigsten Zeit meiner 
Ausbildung als Linguistin, und was ich bei ihm über die Sprache und ihre theoretische Auffassung gelernt habe, wurde dann in der beruflichen Praxis, insbesondere in meiner Übersetzungstätigkeit, immer nur bestätigt. Als kleines Zeichen meiner groß en Dankbarkeit möchte ich ihm dieses Zeugnis meiner Erfahrungen anbieten, in der Hoffnung, er werde in diesen Zeilen weitgehend sich selbst wiederfinden.

7. Laplace, Colette, Théorie du langage et théorie de la traduction: les concepts-clefs de trois auteurs: Kade (Leipzig), Coseriu (Tübingen), Seleskovitch (Paris) (Préface de Jean-Marie Zemb), Didier Érudition (Publié avec le concurs du Centre de Recherches en Traductologie, Université de Paris III ; collection «Traductologie», 8), París, I994. En pág. 298 se fichan 13 trabajos de Coseriu: todos en alemán, salvo dos, en francés. El tratamiento de nuestro autor ocupa la parte segunda (págs. 93-177) y se estructura en dos capítulos. En la cuarta parte, «Convergences», los apartados I.2 y 2 son, respectivamente, «Lexique de la terminologie de Coseriu» $\mathrm{y}$ «Peut-on poser des équivalences notionnelles entre les trois conceptions». Reseña, concentrada en la parte de Coseriu, de Lucian Lazăr, en Studia Universitatis Babeş-Bolyai. Philologia (Cluj-Napoca), XLVI/200I («Număr special dedicat Profesorului Eugenio Coşeriu la aniversaria a 80 de ani»), págs. I45-I49.
8. Ulrich, Mioriţa, Die Sprache als Sache: Primärsprache, Metasprache, Übersetzung. Untersuchungen zum Übersetzen und zur Übersetzbarkeit anhand von deutschen, englischen und vor allem romanischen Materialien, Gunter Narr Verlag (Romanica Monacensia, 49), Tubinga, 1997. En pág. 382 se fichan ro trabajos del maestro, de su maestro; sobresaliente monografía en la que el magisterio de Coseriu se halla por doquier: en la segunda parte, Theorie, cap. I, 5.2, «Coserius Übersetzungstheorie»; cap. II, 3.3.I, «Eugenio Coseriu: Fragestellung und theoretischer Rahmen»; 3.3.5, «Bewertung der Leistung von Coseriu»; y ya fuera de su órbita específica, en el cap. III, I.3.2, «Der Ansatz von Coseriu»; 3. Barchudarov und Coseriu»; en el cap. vI, 2.2.0, «Von Coseriu zu Harris».

9. Morani, Moreno, «Sensum de sensu, verbum e verbo. Riflessioni su teoria e storia della traduzione in margine a uno scritto di Eugenio Coseriu», en Supplemento a Plurilinguismo. Contatti di Lingue e Culture, Io. Studi in memoria di Eugenio Coseriu (a cura di Vincenzo Orioles), Università di Udine, 2003, págs. 317-336. En la primera página, en las notas i y 2, se cita una amplia lista de los estudios del maestro sobre la traducción; el foco se halla en el consabido «Lo erróneo y lo acertado en la teoría de la traducción».

RECIBIDO EN MAYO DE $20 I I$ ACEPTADO EN JUNIO DE 2 OII VERSIÓN FINAL DE MAYO DE 2 OII 